\title{
MACROSCOPIC KINK INSTABILITIES IN TOROIDAL RELATIVISTIC BEAMS*
}

\author{
D. A. Spong, O. C. EldRIDGE $\dagger$ \\ Oak Ridge National Laboratory, Oak Ridge, Tennessee 37830, U.S.A. \\ and \\ T. KAMMASH \\ University of Michigan, Ann Arbor, Michigan, U.S.A.
}

(Received 15 October 1976)

\begin{abstract}
Applications of relativistic beams in confinement and heating of toroidal plasmas have been discussed in recent years. We have examined the interaction of such beams with the shear Alfvén modes of the background plasma and have found a class of low-frequency instabilities which are similar to the hydrodynamic kink instabilities of a current-carrying plasma. However, for the case of a beam-plasma configuration, the spectrum of the Alfvén modes is, in general, complex, which precludes the use of an energy principle. We employ a normal mode approach and have calculated both growth rates and real frequencies for $n=1, m=1,2,3,4$ instabilities for various values of beam energy and $v_{\mathrm{A}} / c$. We assume a cold model for the background plasma, but do not make the MHD ordering $\omega \ll \omega_{c i}$. A fixed boundary model of the beam-plasma system is employed and effects of energy spread in the beam are discussed.
\end{abstract}

\section{INTRODUCTION}

THERE HAS been a continuing interest over the past few years in the use of intense relativistic beams in toroidal fusion configurations such as the Tokamak (HAMMER and PAPAdopoulos, 1975; Mohri et al., 1975; BenFord et al., 1974, Gilad et al., 1974; SwaIn et al, 1975). Some of the motivations behind this application are those of supplementary plasma heating relatively steady-state toroidal currents due to the long classical life-times of high energy electrons; and possibly altered macroscopic stability properties (KNOEPFEL et al., 1976; SPONG et al., 1974; LEE, 1973; LovelACE, 1976). In this paper we wish to examine the third feature in particular.

Several methods have been suggested for generating and sustaining such beams. One is to externally inject a diode-generated beam; the feasibility of such a scheme is now under examination at a number of laboratories. A primary difficulty to be overcome is the achievement of an efficient process for injecting charged particels across strong magnetic field lines. Techniques which have been suggested involve either a momentary disruption of the magnetic surfaces at the instant of injection (GILAD et al., 1974) or a reliance on single particle drifts to carry the beam into the plasma region (BENFORD et al., 1974; SwAIN et al., 1975).

A second method for creating such a beam is the electron runaway phenomenon-which occurs whenever an electric field is applied to a plasma. It has been observed in several Tokamak experiments (SPONG et al., 1974; VLASEKOV et al., 1973; ALIKAEV et al., 1973; AlIKAEV et al., 1975) that in certain regimes of operation (low density, high impurity), runaway-dominated discharges may be produced where a group of relativistic $(1-7 \mathrm{MeV})$ electrons carries a

\footnotetext{
* Research sponsored by the Energy Research and Development Administration under contract with Union Carbide Corporation.

${ }^{\dagger}$ Consultant, University of Tennessee, Knoxville, Tennessee.
} 
substantial portion of the toroidal current. These discharges have indicated a number of interesting features such as anomalous ion heating (ALIKAEV et al., 1975); low levels of externally measured MHD activity (KNOEPFEL et al., to be published; VLASENKOV et al., 1973); high $\beta_{p}$ values, indicating a more efficient kinetic energy storage capability than has been obtained in normal Tokamak discharges (KNOEPFEL et al., to be published; AlIKAEV et al., 1975); and enhanced levels of microwave emission, near $\omega_{c e}$ and its harmonics (CostLy et al., 1974). Producing toroidal relativistic beams by the runaway method has the advantage that the electrons do not have to be injected across field lines, but has the possible disadvantage that one may not have the same degree of control over the beam energy and density as would be the case with a diode-generated beam.

In the following sections we examine the macroscopic stability features of a toroidal relativistic beam-plasma configuration against kink modes. The current density in conventional Tokamak discharges is typically thought to be limited by the onset of external kink instabilities at the Kruskal-Shafranov limit ( $q=$ $r B_{t} / R_{0} B_{\theta}=1$ ). The examination of such instabilities is of importance for toroidal relativistic beams due to the potentially high current densities which may be carried $\left(j \simeq 50 \mathrm{kA} / \mathrm{cm}^{2}\right.$ per $10^{13} \mathrm{~cm}^{-3}$ of beam density). As mentioned above, there have been several observations that the externally measured MHD activity of Tokamak runaway discharges is at a somewhat lower level than in normal discharges (KNOEPFEl et al., to be published; Vlasenkov et al., 1975). Also, a parameter study (SPONG et al., 1974) made of such discharges has suggested that $q$ may be attaining values less than unity near the central region of the discharge (where the runaway beam was assumed to localized) without complete loss of confinement due to kink instabilities. Although it must be kept in mind that such observations are at present somewhat speculative and are in a highly preliminary stage, they provide motivation for examining possible differences between the gross stability features of a Tokamak configuration in which the toroidal current is carried by low energy conduction electrons and of one in which relativistic electrons carry most of the current. Clearly, since the fusion power density in a Tokamak reactor scales inversely as $q^{4}$, there is substantial reason to consider carefully alternative methods of operating Tokamaks which might allow the Kruskal-Shafranov limit to be exceeded.

Kink instabilities in toroidal relativistic beams were first treated by LEE (1973) who performed a normal mode analysis of these instabilities in a cylindrical geometry. The toroidicity was introduced by means of periodic boundary conditions on the perturbed fields. LEE (1975) found that the stability boundary for the beam against $m= \pm 1, n=1$ kink modes corresponded to the condition of closed particles orbits as opposed to the more conventional condition of closed field lines. Since the particle orbits lag slightly behind field lines (due to the finite particle inertia) this condition is somewhat relaxed from the usual $q=1$ limit. However, in order to allow substantial improvement over this limit for moderate sized Tokamaks and magnetic fields, very high beam energies are required and synchrotron losses would be prohibitive.

More recently, LOVELACE (1976) has also treated the problem of kink instabilities in toroidal relativistic beams by means of an energy principle. $\mathrm{He}$ concluded that the stability conditions for kink modes $(m \neq 0, n \neq 0)$ of the beam 
are identical to those of a conventional plasma pinch provided the plasma pressure is replaced with $p(r)=\left(B^{2} / 8 \pi\right) \beta_{t}$ where $\beta_{t}$ is the ratio of the directed beam kinetic energy density to the toroidal magnetic field $\left(B_{t}\right)$ energy density. His result, in contrast to that of LEE (1973), would then indicate that raising the beam kinetic energy makes the system less stable.

In the following analysis, we consider several effects which were not taken into account in the earlier work and which may be of particular relevance to runaway discharges in present sized Tokamaks. One such effect is the high transit frequency of relativistic electrons (i.e. the frequency at which they move around a flux surface); this can typically be on the order of or greater than the ion cyclotron frequency. This condition leads one to consider a model for the background plasma other than the MHD approximation (which assumes $\omega \ll \Omega_{c i}$ ) employed by LEE (1973) and LOVELACE (1976). An analogous situation has been found to occur in mirror-confined hot electron plasma and has been examined by GuEsT et al. (1975) for the case of ballooning modes; the high electron drift frequency was found to have a significant stabilizing influence on such modes.

A second new feature included in this work is the relative stiffness (parallel mass $=\gamma^{3} m_{0}$, perpendicular mass $=\gamma m_{0}$ ) characteristic of high energy electrons towards momentum changes along their direction of propagation. It was recognized some time ago (Bludman et al., 1960) that in the absence of external magnetic fields, this property made relativistic beams much less subject to kink and sausage modes than plasma pinches operating at similar current densities.

Our analysis is based on a normal mode approach; perturbed beam currents are obtained by integrating along the unperturbed particle orbits. The effects of both perturbed electric and perturbed magnetic fields are included in the force equation for the beam. We also do not make the assuinption, used in previous work, that the frequency characterizing these modes is only purely real or purely imaginary. This corresponds to keeping terms of order $k_{\|} v_{\|}$relative to $\omega$ in the dispersion relation. The fact that $\omega$ is in general a complex number prevents the use of an ènergy principle and refiects the fact that the operators involved in the beam equation of motion are not self-adjoint. This feature occurs in any plasma system where there is a net flow of one component relative to a second component. Instabilities which are purely growing or damped in the moving frame ( $\omega$ is imaginary) appear with a superimposed oscillation in the laboratory frame ( $\omega$ is complex).

Our analysis proceeds according to the following outline. We first discuss the physical model, particle orbits, and perturbed field equations. Next, the perturbed currents for the plasma and beam species are calculated. Substituting these into the perturbed field equations and invoking the appropriate boundary conditions results in a dispersion relation. This is then solved for the cases with no beam (plasma normal modes) and with a beam. In the latter, unstable roots are found and examined with respect to such parameters as beam energy, rotational transform, poloidal mode number and $v_{\mathrm{A}} / \mathrm{c}$.

\section{PHYSICAL MODEL, PARTICLE ORBITS, AND FIEID}

The model assumed in this work that of a cylindrical relativistic electron beam immersed in a cold background plasma and surrounded by a conducting wall. A 
constant magnetic field is applied along the axis of the cylinder; it is assumed that the beam and plasma do not significantly modify this magnetic field, i.e. there is no net diamagnetism or paramagnetism. The effect of toroidicity is introduced by periodic boundary conditions on the perturbed fields. Helical perturbations of the form $\exp [i(m \theta+n \zeta-\omega t)], \zeta=z / R_{0}(n, m$ are integers) are considered. The use of a cold plasma model is justified by the long perpendicular wavelength of the instabilities under consideration. This wavelength is on the order of the plasma minor radius whereas electron and ion Larmor radii are, at most, a few millimeters. Thus, $k_{\perp} \rho_{i}$ and $k_{\perp} \rho_{e} \ll 1$ and finite Larmor radius corrections for the background plasma are small.

Since a uniform axial current density is assumed, $B_{\theta}$ and $q$ are given by the foilowing:

$$
\begin{gathered}
B_{\theta}(r)=\frac{4 \pi}{c r} \int_{0}^{r} J(r) r \mathrm{~d} r=\left\{\begin{array}{ll}
2 I r / c a^{2} & 0<r<a \\
2 I / c r & r>a
\end{array},\right. \\
q(r)=\frac{r B_{z}}{R_{0} B_{\theta}}=\left\{\begin{array}{cc}
q(a) & 0<r<a \\
r^{2} B_{z} c / 2 R_{0} I & r>a
\end{array}\right.
\end{gathered}
$$

where $a=$ radius of the current channel.

In Cartesian coordinates $B_{\theta}$ may be expressed as

$$
B_{x}=-\frac{y B_{z}}{q R_{0}} \quad B_{y}=\frac{x B_{z}}{q R_{0}},
$$

The unpertiribed relâtivistic orbits are then given by the components of the force equation,

$$
\begin{aligned}
& \frac{\mathrm{d}}{\mathrm{d} t}\left(\gamma v_{x}\right)=\Omega_{e} v_{y}-\frac{\Omega_{e} x}{q R_{0}} v_{z}, \\
& \frac{\mathrm{d}}{\mathrm{d} t}\left(\gamma v_{y}\right)=-\Omega_{e} v_{x}-\frac{\Omega_{e} y}{q R_{o}} v_{z}, \\
& \frac{\mathrm{d}}{\mathrm{d} t}\left(\gamma v_{z}\right)=\frac{\Omega_{e}}{q R_{o}}\left(x v_{x}+y v_{y}\right) \simeq 0,
\end{aligned}
$$

where

$$
\Omega_{e}=\frac{e B_{z}}{m_{0} c}
$$

Since no unperturbed electric field is assumed, $\gamma=$ constant. Defining $v^{ \pm}=$ $v_{x} \pm i v_{y}, x^{ \pm}=x \pm i y$ and assuming $x^{ \pm}=x_{\perp} \mathrm{e}^{ \pm i \omega t}$ one obtains from (4) the following equation for $\omega$ :

$$
\gamma \omega^{2}-\Omega_{e} \omega-\frac{\Omega_{e} v_{z}}{q R_{o}}=0
$$




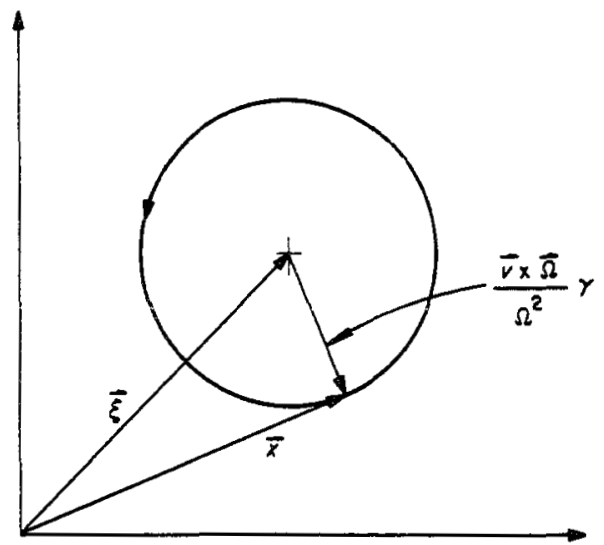

FIG. 1.-Guiding center and gyromotion for a relativistic electron in a magnetic field.

which has the two roots:

$$
\begin{aligned}
& \omega_{+}=\frac{\Omega_{e}}{2 \gamma}\left[1+\sqrt{1+4 v_{z} \gamma / q R_{0} \Omega_{e}}\right] \simeq \frac{\Omega_{e}}{\gamma}, \\
& \omega_{-}=\frac{\Omega_{e}}{2 \gamma}\left[1-\sqrt{1+4 v_{z} \gamma / q R_{0} \Omega_{e}}\right] \simeq-\omega_{b r}\left(1-\frac{2 \gamma \omega_{b r}}{\Omega_{e}}\right),
\end{aligned}
$$

where $\omega_{b r}=v_{z} / q R_{0}=$ transit or bounce frequency.

$\omega_{+}$and $\omega_{-}$are approximately the gyrofrequency of a relativistic electron and the frequency corresponding to motion along the field lines, respectively. $v^{ \pm}$may be readily calculated by separating out the guiding center motion as indicated in the following equation and in Fig. 1:

$$
\xi=\mathbf{x}+\frac{\mathbf{v} \times \boldsymbol{\Omega}}{\Omega^{2}} \gamma=\mathbf{x}+(\mathbf{v} \times \hat{b}) / \omega_{+}
$$

Here $\xi=$ position of the guiding center. Writing $\xi$ in terms of its Cartesian coordinates and taking time derivates results in the equations

$$
\begin{aligned}
& \frac{\mathrm{d} \xi_{x}}{\mathrm{~d} t}=v_{x}+\frac{1}{\omega_{+} \gamma}\left[-\Omega_{e} v_{x}-\frac{\Omega_{e} y}{q R_{0}} v_{z}\right], \\
& \frac{\mathrm{d} \xi_{y}}{\mathrm{~d} t}=v_{y}-\frac{1}{\omega_{+} \gamma}\left[\Omega_{e} v_{y}-\frac{\Omega_{e} x}{q R_{0}} v_{z}\right] .
\end{aligned}
$$

Combining equations (8), (7) and (5), one obtains the following equation for $\xi^{ \pm}=\xi_{x} \pm i \xi_{y}$ :

$$
\frac{\mathrm{d} \xi^{ \pm}}{\mathrm{d} t}= \pm \frac{i \Omega_{e} v_{z}}{\omega_{+} \gamma q R_{0}} \xi^{ \pm} \approx \mp i \omega_{-} \xi^{ \pm}
$$

Thus $\xi^{ \pm}=\xi_{\perp} e^{ \pm i(\phi-\omega t)}$ where $\phi$ is an arbitrary phase factor. 
The transverse equations of motion given in (4) may now be written in terms of $\xi$, the guiding center coordinate, as

$$
\begin{aligned}
& \frac{\mathrm{d}}{\mathrm{d} t}\left(\gamma v_{x}\right)=\Omega_{e} v_{y}-\frac{\Omega_{e} v_{z}}{q R_{0}}\left(\xi_{x}-v_{y} / \omega_{+}\right), \\
& \frac{\mathrm{d}}{\mathrm{d} t}\left(\gamma v_{y}\right)=-\Omega_{e} v_{x}-\frac{\Omega_{e} v_{z}}{q R_{0}}\left(\xi_{\mathrm{y}}+v_{x} / \omega_{+}\right) .
\end{aligned}
$$

These may be combined into an equation for $v^{ \pm}=v_{x} \pm i v_{y}$ :

$$
\frac{\mathrm{d} v^{ \pm}}{\mathrm{d} t}=\mp i \omega_{+} v^{ \pm}+\omega_{+} \omega_{-} \xi^{ \pm} .
$$

Substituting (9) for $\xi^{ \pm}$one the obtains a solution for $v^{ \pm}$,

$$
v=v_{\perp} \mathrm{e}^{ \pm i\left(\psi-\omega_{+} t\right)} \mp \frac{i \omega_{-} \omega_{+} \xi_{\perp} e^{i \phi}}{\omega_{+}-\omega_{-}}\left[\mathrm{e}^{\mp i \omega_{-} t}-\mathrm{e}^{\mp i \omega_{+} t}\right] .
$$

As may be seen, (12) has components corresponding to both the gyromotion (at $\omega_{+}$) and the motion along field lines (at $\omega_{-}$). Note that the frequency $\omega_{-}$is dependent on the energy $\gamma m_{0} c^{2}$; this is related to the fact that the electrons lag slightly behind field lines due to their finite mass. This dependence will be of importance in the Vlasov treatment which is given in Appendix A. A spreading out in the energy distribution of the relativistic electrons is related to a spreading out in the position of the particle orbits away from field lines.

We now turn to the electromagnetic field equations which are employed in this instability analysis. For perturbed fields with the dependence $\exp [i(m \theta+n \xi-\omega t)]$, the components of Maxwell's curl $E$ and curl $B$ equations (in cylindrical geometry) result in the following six equations:

$$
\begin{gathered}
\frac{1}{\rho} \frac{\partial}{\partial \rho}\left(\rho E_{\theta}\right)-\frac{i m}{\rho} E_{r}=i B_{z}, \\
\frac{m}{\rho} E_{z}-n_{\|} E_{\theta}=B_{r}, \\
n_{\|} E_{r}+i \frac{\partial E_{z}}{\partial \rho}=B_{\theta} \\
\frac{i}{\rho} \frac{\partial}{\partial \rho}\left(\rho B_{\theta}\right)+\frac{m}{\rho} B_{r}=E_{z}+\frac{4 \pi i J_{z}}{\omega}, \\
-\frac{m}{\rho} B_{z}+n_{\|} B_{\theta}=E_{r}+\frac{4 \pi i J_{r}}{\omega}, \\
-n_{\|} B_{r}-i \frac{\partial B_{z}}{\partial \rho}=E_{\theta}+\frac{4 \pi i J_{\theta}}{\omega},
\end{gathered}
$$

where $\rho=r \omega / c$ and $n_{\|}=n c / R_{0} \omega$.

In the following instability analysis, these equations are combined in the following manner: $B_{r}$ and $B_{\theta}$ from (14) and (15) are substituted into (17) and (18) 
to obtain equations for $E_{r}$ and $E_{\theta}$ and terms of $E_{z}, B_{z}, J_{r}$ and $J_{\theta}$. These are then substituted into (13) and (16) resulting in two differential equations for the perturbed longitudinal fields $E_{z}$ and $B_{z}$ :

$$
\begin{gathered}
\nabla_{\perp}^{2} B_{z}+\left(1-n_{\|}^{2}\right) B_{z}=\frac{4 \pi}{\omega \rho}\left[i \omega J_{r}-\frac{\partial}{\partial \rho}\left(\rho J_{\theta}\right)\right], \\
\nabla_{\perp}{ }^{2} E_{z}+\left(1-n_{\|}^{2}\right) E_{z}=-\frac{4 \pi i}{\omega}\left\{\left(1-n_{\|}^{2}\right) J_{z}+\frac{n_{\|}}{\rho}\left[i \frac{\partial}{\partial \rho}\left(\rho J_{r}\right)-m J_{\theta}\right]\right\},
\end{gathered}
$$

where

$$
\nabla_{\perp}^{2}=\frac{1}{\rho} \frac{\partial}{\partial \rho} \rho \frac{\partial}{\partial \rho}-\frac{m^{2}}{\rho^{2}}
$$

The remaining task is to determine the perturbed currents $\delta J$ in terms of the perturbed fields $E_{z}$ and $B_{z}$; this is done in Section 3. Since $\delta J$ will generally depend on both longitudinal and transverse components of $\mathbf{E}$ and $\mathbf{B}$, it is necessary to use (14), (15), (17) and (18) to express $\delta J$ in terms of $E_{z}$ and $B_{z}$. Once $J_{r}$ and $J_{\theta}$ are specified, the transverse electric fields are obtained (in terms of $E_{z}$ and $B_{z}$ ) by solving the equations

$$
\begin{aligned}
& \frac{4 \pi i}{\omega} J_{r}+\left(1-n_{\|}^{2}\right) E_{r}=-\frac{m}{\rho} B_{z}+i n_{\|} \frac{\partial E_{z}}{\partial \rho}, \\
& \frac{4 \pi i}{\omega} J_{\theta}+\left(1-n_{\|}^{2}\right) E_{\theta}=-\frac{m n_{\|}}{\rho} E_{z}-i \frac{\partial B_{z}}{\partial \rho} .
\end{aligned}
$$

When $\mathbf{j}=0$ (no piasma or beam), the two equations reduce to the usual waveguide TM and TE equations as given for example, in JoHnson (1965). Once two coupled eigenmode equations are obtained for $E_{z}$ and $B_{z}$ from (19) and (20), solution and application of appropriate boundary conditions results in a dispersion relation. This will be worked out in Section 4 for a fixed boundary model.

In the previous work on kink instabilities the coupling between (19) and (20) has been neglected. LOVELACE (1976) assumes $\delta E \cdot \mathbf{B}=0$ whereas LeE (1973) neglects all perturbed electric fields. These approximations are related to the assumption of a highly conducting plasma and result in consideration only of (19) (or its equivalent in terms of vector potential $\delta A_{\theta}$ ) with $J_{r}$ and $J_{\theta}$ expressed in terms of $\delta B_{z}$ (or $\delta A_{\theta}$ ).

\section{PERTURBED CURRENTS}

\section{Background plasma}

The dynamics of the electrons and ions in the background plasma are described by the force equations

$$
\begin{gathered}
\frac{\mathrm{d} v_{e, i}^{ \pm}}{\mathrm{d} t}=\mp i \Omega_{e, i} v_{e, i}^{ \pm}-\frac{v_{z e, i} \Omega_{e, i} x_{e, i}^{ \pm}}{q R_{0}}+\frac{e}{m_{e, i}} E^{ \pm}, \\
\frac{\mathrm{d} v_{z e, i}}{\mathrm{~d} t}=\frac{\Omega_{e, i}}{q R_{0}} \frac{\mathrm{d}}{\mathrm{d} t}\left(\frac{r^{2}}{2}\right)+\frac{e}{m_{e, i}} E_{z} .
\end{gathered}
$$


The orbits of the electrons and ions are

$$
\begin{gathered}
v_{e, i}^{ \pm}=v_{\perp} \mathrm{e}^{ \pm i\left(\psi-\omega_{+} t\right)} \mp \frac{i \omega_{+} \omega_{-}}{\omega_{+}+\omega_{-}} \xi_{\perp} \mathrm{e}^{ \pm i \theta_{0}}\left[\mathrm{e}^{ \pm i \omega_{-} t}-\mathrm{e}^{ \pm i \omega_{+} t}\right], \\
v_{e, i}^{z}=\frac{\Omega_{e, i} r^{2}}{2 q R_{0}}+v_{\|},
\end{gathered}
$$

where $v_{\|}, v_{\perp}, \theta_{0}, \xi_{\perp}=$ initial conditions.

$\omega_{+}$and $\omega_{-}$are the non-relativistic analogues of the frequencies defined in (6). Since the analysis here is for a cylinder with a constant magnetic field, it will not include certain effects which occur in a toroidal geometry. These include the trapping of electrons and ions which mirror on the high field side of the cross section. Such effects will be ignored since they are of importance for kilocycle frequencies, but not for megacycle frequencies near the Alfvén transit frequency. The guiding center for the above orbits is defined by

$$
\xi_{x}=x+\frac{v_{y}}{\omega_{+}}, \quad \xi_{y}=y-\frac{v_{x}}{\omega_{+}}
$$

and has the time dependence $\xi^{ \pm}=\xi_{\perp} e^{ \pm i\left(\theta-\omega_{-} t\right)}$.

Given the above unperturbed orbits, one may calculate perturbed velocities by retaining the perturbed electric field terms in (23) and (24). The following dependence has been assumed for the perturbed fields:

$$
\begin{aligned}
E^{ \pm} & =E_{x} \pm i E_{y} \\
& =\left[\varepsilon_{r}(r) \pm i \varepsilon_{\theta}(r) \exp i\left[(m \pm 1) \theta+n z / R_{0}-\omega t\right]\right.
\end{aligned}
$$

Since only long wavelength modes are of interest in this work, it is assumed that $\varepsilon_{r}$ and $\varepsilon_{\theta}$ are slowly varying functions of $r$ (in comparison to a gyroradius). Such an ordering implies that the $r$ and $\theta$ positions of plasma electrons and ions are essentially constant over the time scale of the instability. The only variation in position allowed is that due to gyromotion, the bounce motion is neglected since it is on a much slower time scale than that characterizing the instability. Under the above approximations, the perturbed plasma currents are given by

$$
4 \pi J_{e, i}^{ \pm}=\frac{i \omega_{p e, i}^{2} E^{ \pm}}{\omega \mp \Omega_{e, i}}, \quad 4 J_{z e, i}=-\frac{i \omega_{p e, i}^{2} E_{z}}{\omega} .
$$

In the case of electrons, we neglect $\omega$ in comparison with $\Omega_{e}$; however, as mentioned earlier, $\Omega_{i}$ is retained in comparison to $\omega$ since these frequencies are not necessarily far separated.

Relativistic beam perturbed currents

The starting point for calculating the perturbed beam currents is the relativistic 
force equation; the Cartesian components of $t$ equation are given by

$$
\begin{aligned}
& \frac{\mathrm{d}}{\mathrm{d} t}\left(\gamma v_{x}\right)=\Omega_{e} v_{y}-\frac{\Omega_{e} v_{z} x}{q R_{0}}-\frac{e}{m_{0}}\left(. \quad-\frac{v_{y} B_{z}-v_{z} B_{y}}{c}\right), \\
& \frac{\mathrm{d}}{\mathrm{d} t}\left(\gamma v_{y}\right)=-\Omega_{e} v_{x}-\frac{\Omega_{e} v_{z} y}{q R_{0}}-\frac{e}{m_{0}}\left(+\frac{v_{z} B_{x}-v_{x} B_{z}}{c}\right), \\
& \frac{\mathrm{d}}{\mathrm{d} t}\left(\gamma v_{z}\right)=-\frac{\Omega_{e}}{q R_{0}}\left(x v_{x}+y v_{y}\right)-\frac{\left(E_{z}+\frac{v_{x} B_{y}-v_{y} B_{x}}{c}\right) .}{c}
\end{aligned}
$$

Here the fields $E_{x}, B_{x}, \ldots$ are perturbed fiel the equilibrium fields are contained in $\Omega_{e}$ and $q$. In the following analysis e terms arising from transverse beam velocities crossed into the longitudinal transverse perturbed magnetic fields are neglected. Such terms are expecte, , be down by an order $(q A)^{-1}$ $(A=$ aspect ratio) from those retained. We ill also average the perturbed equations of motion over the gyromotion, i.e. $\quad$ drift approximation is used. A final assumption is that all non-linear terms $\mathrm{r}$.

It will be recalled from Section 1 that the be neglected.

perturbed guiding center orbits are

$$
\begin{aligned}
v_{x} & =-\omega_{b r} y, \\
v_{y} & =\omega_{b r} x \\
\frac{\mathrm{d}}{\mathrm{d} t}\left(\gamma v_{z}\right) & =-\frac{\Omega_{e}}{q R_{0}}\left(-\omega_{b r} x y \quad \omega_{b r} x y\right)=0 .
\end{aligned}
$$

The Cartesian coordinates of the unperturbec bits are then given by

$$
\begin{aligned}
& x=r \cos \left(\theta_{0}+c\right. \\
& y=r \sin \left(\theta_{0}+a\right. \\
& z=z_{0}+v_{\|} t .
\end{aligned}
$$

The first order perturbed beam velocities are

$$
\begin{aligned}
v_{x} & =-\omega_{b r} y-\frac{e}{m_{0} \Omega_{e}}\left(E \quad \frac{v_{z}}{c} B_{x}\right), \\
v_{y} & =\omega_{b r} x+\frac{e}{m_{0} \Omega_{e}}\left(E_{x}-\quad \Xi B_{y}\right), \\
\frac{\mathrm{d}}{\mathrm{d} t}\left(\gamma v_{z}\right) & =-\frac{e}{m_{0}} E_{z} .
\end{aligned}
$$

Also, from the relativistic energy equation, 0 has

$$
\frac{\mathrm{d} \gamma}{\mathrm{d} t}=-\frac{e}{m_{0} c^{2}} v_{z}
$$


The perturbed velocities in the longitudinal and transverse directions are then obtained by integrating equations (33) and (34) with respect to time over the unperturbed orbits. First, for the perturbed velocity in the $z$ direction,

$$
\delta\left(\gamma v_{z}\right)=\gamma \delta v_{z}+v_{\|} \delta \gamma=-\frac{e}{m_{0}} \int_{-\infty}^{t} \mathrm{~d} t^{\prime} E_{z}\left(t^{\prime}\right)=-\frac{i e E_{z}}{m_{0}\left[\omega-(m+n q) \omega_{b r}\right]}
$$

is obtained.

Also, integrating the energy equation (34) results in

$$
\delta \gamma=-\frac{i e v_{\|} E_{z}}{m_{0} c^{2}\left[\omega-(m+n q) \omega_{b r}\right]} .
$$

Combining (35) and (36) then gives the perturbed longitudinal velocity,

$$
\delta v_{x}=\frac{-i e E_{z}}{m_{0} \gamma^{3}\left[\omega-(m+n q) \omega_{b r}\right]} .
$$

In order to obtain the perturbed transverse velocities from equations (33) it is necessary to first calculate the perturbed positions; these may be found by integrating the first two equations of (33). Written in terms of $x^{ \pm}$and $v^{ \pm}$, these are given by

$$
v^{ \pm}=\frac{\mathrm{d} x^{ \pm}}{\mathrm{d} t}= \pm i \omega_{b r} x^{ \pm}-\frac{e}{m_{0} \Omega_{e}}\left(\mp i E^{ \pm}+\frac{v_{z}}{c} B^{ \pm}\right) .
$$

Equation (38) has the solution

$$
x^{ \pm}=r e^{ \pm i\left(\theta_{0}+\omega_{b r}\right)}+\delta x^{ \pm} .
$$

The perturbed position $\delta x^{ \pm}$is obtained by integrating over the unperturbed orbits and is given by

$$
\delta x^{ \pm}= \pm \frac{e\left(E^{ \pm} \pm i V_{z} B^{ \pm} / c\right)}{m_{0} \Omega_{e}\left[\omega-(m+n q) \omega_{b r}\right]}
$$

Substituting the above result back into (38) leads to the desired transverse perturbed velocities,

$$
v^{ \pm}=v_{\perp} \mathrm{e}^{ \pm i\left(\theta_{0}+\omega_{\mathrm{sr}} t\right)}+\delta v^{ \pm}
$$

where

$$
\delta v^{ \pm}=\mp \frac{i e}{m_{0} \Omega_{e}}\left(E^{ \pm} \pm i v_{z} B^{ \pm} / c\right)\left[1 \mp \frac{\omega_{b r}}{\omega-(m+n q) \omega_{b r}}\right] .
$$

Now that the perturbed velocities have been calculated, perturbed beam currents may be found. The perturbed distribution function is given by

$$
\delta f=F_{0}\left(v_{\perp}, v_{\|}, r\right)-\delta v_{\perp} \frac{\partial F_{0}}{\partial v_{\perp}}-\delta v_{\|} \frac{\partial F_{0}}{\partial v_{\|}}-\delta r \frac{\partial F_{0}}{\partial r} .
$$

For the case of a beam of relativistic electrons with uniform velocity near the speed of light and no transverse thermal energy, an appropriate distribution function is $F_{0}=\delta\left(v_{z}-v_{0}\right) \delta\left(v_{\perp}-r \omega_{b r}\right)$ where $v_{0} \simeq c$. The perturbed currents in this 
case are given by

$$
\begin{gathered}
4 \pi J^{ \pm}= \pm \frac{i \omega_{p b}{ }^{2}}{\Omega_{e}}\left(E^{ \pm} \pm i v_{z} B^{ \pm} / c\right)\left[1 \mp \frac{\omega_{-}}{\omega-(m+n q) \omega_{b r}}\right] \\
4 \pi J_{z}=\frac{i \omega_{p b}{ }^{2}}{\gamma^{3}} \frac{E_{z}}{\omega-(m+n q) \omega_{b r}}+\frac{i v_{z}}{\Omega_{e}} \frac{\partial}{\partial r}\left(\omega_{p b}{ }^{2}\right) \frac{E_{\theta}+B_{r}}{\omega-(m+n q) \omega_{b r}}
\end{gathered}
$$

where

$$
\omega_{p b}^{2}=\frac{4 \pi n_{b} e^{2}}{m_{0}} .
$$

The final term in (44) arises from the integral $\int 4 \pi e \mathrm{~d}^{3} v \delta r_{z} \partial F_{0} / \partial r$. A uniform density profile is assumed in the following analysis so that this last term of $J_{z}$ will be absent. If one wished to examine the case in which the beam density was still uniform, but localized within the plasma at a radius $r_{b}$, then the final term of (44) would contribute a Dirac delta function $\delta\left(r-r_{b}\right)$ to the perturbed $J_{z}$. This is physically due to the perturbed surface current caused by the gross motion of the beam boundary as it is undergoing displacement. In this situation, boundary conditions on the perturbed fields at $r=r_{b}$ must be derived by multiplying the two coupled equations (19) and (20) by $r \mathrm{~d} r$ and integrating over the layer $r_{b}-\varepsilon$ to $r_{b}+\varepsilon$ with $\varepsilon \rightarrow 0$.

For the case in which the relativistic beam has an energy spread, a Vlasov approach must be employed in calculating perturbed currents. This approach is presented in Appendix A.

\section{DISPERSION RELATION DERIVATION}

A dispersion relation is derived for the case of a fixed boundary beam-plasma model (beam and plasma extend all the way to a conducting wall). For simplicity, the perturbed beam currents derived in Section 3 will be used; the derivation could readily be generalized to the case of the Vlasov beam model given in Appendix A. The derivation is made according to the following outline. First, the perturbed currents for the three species present, electrons, ions, beam electrons, are substituted into the field equations of Section 2. Next, these equations are manipulated until two coupled equations for the perturbed fields $E_{z}$ and $B_{z}$ are obtained. Finally, a method for decoupling these equations is used and a dispersion relation is obtained by requiring the appropriate boundary conditions to be satisfied by $E_{z}$ and $B_{z}$.

Adding up the perturbed currents for the three species as given by (29), (38) and (39) results in the following currents:

$$
\begin{gathered}
\frac{4 \pi J_{r}}{\omega}=\frac{i \omega_{p i}^{2}}{\omega^{2}-\Omega_{i}^{2}} E_{r}-\frac{\omega_{p i}^{2} \omega}{\left(\omega^{2}-\Omega_{i}^{2}\right) \Omega_{i}} E_{\theta}-\frac{i \omega_{p b}^{2} \omega_{b r}\left[E_{r}-v_{z} B_{\theta} / c\right]}{\left[\omega-(m+n q) \omega_{b r}\right]}, \\
\frac{4 \pi J_{\theta}}{\omega}=\frac{i \omega_{p i}^{2}}{\omega^{2}-\Omega_{i}^{2}} E_{\theta}+\frac{\omega_{p i}^{2} \omega}{\left(\omega^{2}-\Omega_{i}^{2}\right) \Omega_{i}} E_{r}-\frac{i \omega_{p b}^{2} \omega_{b r}\left[E_{\theta}+v_{z} B_{r} / c\right]}{\omega \Omega_{e}\left[\omega-(m+n q) \omega_{b r}\right]}, \\
\frac{4 \pi J_{z}}{\omega}=\frac{i \omega_{p e}^{2}}{\omega^{2}} E_{z}+\frac{i \omega_{p b}^{2}}{\omega \gamma^{3}} \frac{E_{z}}{\left[\omega-(m+n q) \omega_{b r}\right]}
\end{gathered}
$$


The field (14) and (15) are use $\quad$ r $B_{r}$ and $B_{\theta}$; also, the following quantities are defined:

$$
\begin{gathered}
Q=\frac{\omega_{b r} \omega_{p b}^{2}}{{ }^{2}\left[\omega-(m+n q) \omega_{b r}\right]}, \\
=\frac{\omega \omega_{p i}^{2}}{\Omega_{i}\left(\omega^{2}-\Omega_{i}^{2}\right)}, \\
=1-\frac{\omega_{p i}^{2}}{\omega^{2}-\Omega_{i}^{2}}, \\
P=1-\frac{\omega_{p b}^{2}}{\omega \gamma^{3}\left[\omega-(m+n q) \omega_{b r}\right]}
\end{gathered}
$$

The perturbed currents (45)-(4) nay then be written in the form

$$
\begin{aligned}
& \frac{4 \pi i J_{r}}{\omega}=\left[\begin{array}{ll}
S-1 & \left.1-n_{\|}\right) Q
\end{array}\right] E_{\mathrm{r}}-i D E_{\theta}-i Q \frac{\partial E_{z}}{\partial \rho}, \\
& \frac{4 \pi i J_{\theta}}{\omega}=\left[\begin{array}{ll}
S-1 & \left.1-n_{\sharp}\right) Q
\end{array}\right] E_{\theta}+i D E_{r}+i Q \frac{\partial E_{z}}{\partial \rho}, \\
& \frac{4 \pi i J_{z}}{\omega}=\left(\begin{array}{ll}
P-1 & . .
\end{array}\right.
\end{aligned}
$$

The transverse perturbed electr ields, $E_{r}$ and $E_{\theta}$, are now obtained in terms of $E_{z}$ and $B_{z}$ by substituting $J_{r}: J_{\theta}$, as given above, into (21) and (22). It is convenient to define the follow: quantities:

$$
\begin{aligned}
& \varepsilon_{1}=\imath {\left[S-n_{\|}{ }^{2}+\left(1-n_{\|}\right) Q\right], } \\
& \varepsilon=G^{-1} D\left(n_{\|}+Q\right), \\
& \varepsilon_{3}=1+G^{-2}\left.n_{\|}+Q\right)\left[S-n_{\|}{ }^{2}+\left(1-n_{\|}\right) Q\right], \\
& \varepsilon_{4}=n_{\|} D G^{-1} \\
& G=\left[\begin{array}{ll}
\varepsilon & \left.\eta_{\|}^{2}+\left(1-n_{\|}\right) Q\right]^{2}-D^{2} .
\end{array}\right.
\end{aligned}
$$

$E_{r}$ and $E_{\theta}$ are then obtained, a

$$
\begin{gathered}
E_{\mathrm{r}}=\frac{i}{n_{\|}}\left(\varepsilon_{3}-1\right) \\
E_{\theta}=-i \varepsilon_{1} \frac{\partial B_{z}}{\partial \rho}-\quad-\left(\varepsilon_{3}-1\right) E_{z}+\varepsilon_{2} \frac{\partial E_{z}}{\partial \rho}+\frac{i m}{\rho n_{\|}} \varepsilon_{4} B_{z} .
\end{gathered}
$$

Substituting the above equatior nto (13) results in the equation

$$
\varepsilon_{1} \nabla_{\perp}^{2}+B_{z}+i \varepsilon_{2} \nabla_{\perp}^{2} E_{z}=0
$$

where

$$
\nabla_{\perp} \frac{1}{\rho} \frac{\mathrm{d}}{\mathrm{d} \rho}\left(\rho \frac{\mathrm{d}}{\mathrm{d} \rho}\right)-\frac{m^{2}}{\rho^{2}} .
$$


This is the first of two coupled radial eigenvalue equations whose eigenvalues determine the growth rates of the unstable modes. Combining (14), (15) and (16) with equations (60) and (61) for $E_{r}$ and $E_{\theta}$ then results in the second of the two coupled equations,

$$
\varepsilon_{3} \nabla_{\perp}^{2} E_{z}+P E_{z}-i \varepsilon_{4} \nabla_{\perp}^{2} B_{z}=0 .
$$

In order to examine stability, it is necessary to find eigenvalues of the coupled system of (62) and (63). These may be put into a somewhat more convenient form by multiplying (62) by $-\varepsilon_{3} / \varepsilon_{2}$, adding to (63), and then multiplying (63) by $-i \varepsilon_{1} / \varepsilon_{4}$ and adding to (62). This results in the two coupled equations

$$
\begin{aligned}
& \left(\nabla_{\perp}^{2}+T_{B}^{2}\right) B_{z}=T_{B E}^{2} E_{z}, \\
& \left(\nabla_{\perp}^{2}+T_{E}^{2}\right) E_{z}=T_{E B}^{2} B_{z},
\end{aligned}
$$

where

$$
\begin{aligned}
T_{\mathrm{B}}^{2} & =\varepsilon_{3} /\left(\varepsilon_{1} \varepsilon_{3}-\varepsilon_{2} \varepsilon_{4}\right), \\
T_{E}^{2} & =\varepsilon_{1} P /\left(\varepsilon_{1} \varepsilon_{3}-\varepsilon_{2} \varepsilon_{4}\right), \\
T_{B E}^{2} & =i P \varepsilon_{2} /\left(\varepsilon_{1} \varepsilon_{3}-\varepsilon_{2} \varepsilon_{4}\right), \\
T_{E B}{ }^{2} & =-i \varepsilon_{4} /\left(\varepsilon_{1} \varepsilon_{3}-\varepsilon_{2} \varepsilon_{4}\right) .
\end{aligned}
$$

A technique for decoupling equations of the form (64) and (65) has been given in JoHNSON (1965). The solutions are

$$
\begin{aligned}
& E_{z}=\frac{Z_{1} \dot{\psi}_{2}-Z_{2} \dot{\psi}_{1}}{Z_{1}-Z_{z}}, \\
& B_{z}=\frac{\psi_{1}-\psi_{2}}{Z_{1}-Z_{2}}
\end{aligned}
$$

where

$$
\begin{aligned}
\psi_{1} & =A_{1} J_{m}\left(T_{1} \rho\right), \\
\psi_{2} & =A_{2} J_{m}\left(T_{2} \rho\right), \\
T_{1,2}^{2} & =T_{E}^{2}-Z_{1,2} T_{B E}^{2}, \\
Z_{1,2} & =\frac{T_{E}^{2}-T_{B}^{2}}{2 T_{B E}^{2}}\left[1 \pm \sqrt{1+\frac{4 T_{B E}^{2} T_{E B}^{2}}{\left(T_{E}^{2}-T B^{2}\right)^{2}}}\right] .
\end{aligned}
$$

$A_{1}$ and $A_{2}$ are constants which are determined by the boundary conditions.

As mentioned earlier, in the fixed boundary model both the beam and plasma extend all the way to the wall and there is no vacuum region in between. The wall is assumed to be perfectly conducting, so that the following requirements must be satisfied by the perturbed fields:

$$
E_{z}\left(\rho_{a}\right)=E_{\theta}\left(\rho_{a}\right)=B_{r}\left(\rho_{a}\right)=0,
$$

where $\rho_{a}=a \omega / c, a=$ shell radius. 
Setting $E_{z}$ equal to zero at the boundary (using equations 66 ) leads to the equation

$$
A_{1}=\frac{Z_{1}}{Z_{2}} A_{2} \frac{J_{m}\left(T_{2} \rho_{a}\right)}{J_{m}\left(T_{1} \rho_{a}\right)}
$$

Next, using (61) and setting $E_{\theta}$ to zero at $\rho=\rho_{a}$ leads to the equation

$$
-\left.i \varepsilon_{1} \frac{\partial B_{z}}{\partial \rho}\right|_{r=a}+\left.\varepsilon_{2} \frac{\partial E_{z}}{\partial \rho}\right|_{r=a}+\left.\frac{i m}{\rho_{a} n_{\|}} \varepsilon_{4} B_{z}\right|_{r=a}=0
$$

Substituting (66) and combining with (69) results in the desired dispersion relation,

$$
Z_{2} T_{2}\left(i \varepsilon_{1}+\varepsilon_{2} Z_{1}\right) \frac{J_{m}{ }^{\prime}\left(\rho_{a} T_{2}\right)}{J_{m}\left(\rho_{a} T_{2}\right)}-Z_{1} T_{1}\left(i \varepsilon_{1}+\varepsilon_{2} Z_{2}\right) \frac{J_{m}{ }^{\prime}\left(\rho_{a} T_{1}\right)}{J_{m}\left(\rho_{a} T_{1}\right)}+\frac{i m \varepsilon_{4}}{\rho_{a} n_{\|}}\left(Z_{1}-Z_{2}\right)=0
$$

\section{NORMAL PLASMA ALFVÉN MODES}

Before examining the unstable spectra (presented in Section 6), the roots of the fixed boundary dispersion relation are obtained (equation (66)) with the relativistic beam density set equal to zero. It can be shown that in this case only

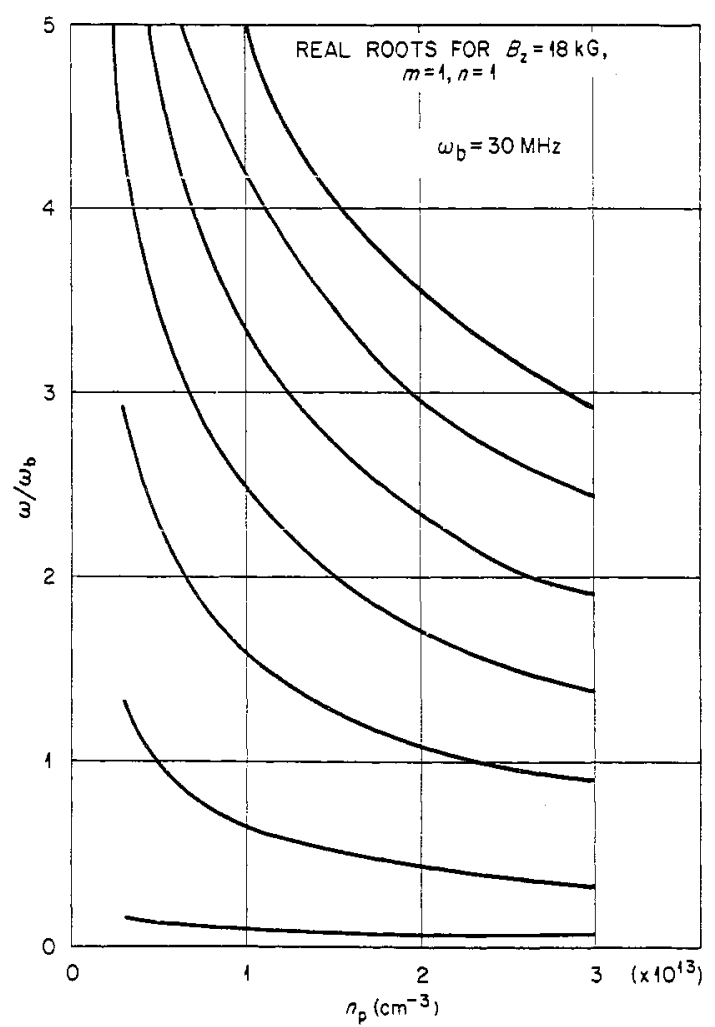

FIG. 2.-Roots of the fixed boundary dispersion relation vs. $n_{\mathrm{p}}$ with $b_{B}=0$ and at a fixed $B_{z}$. 


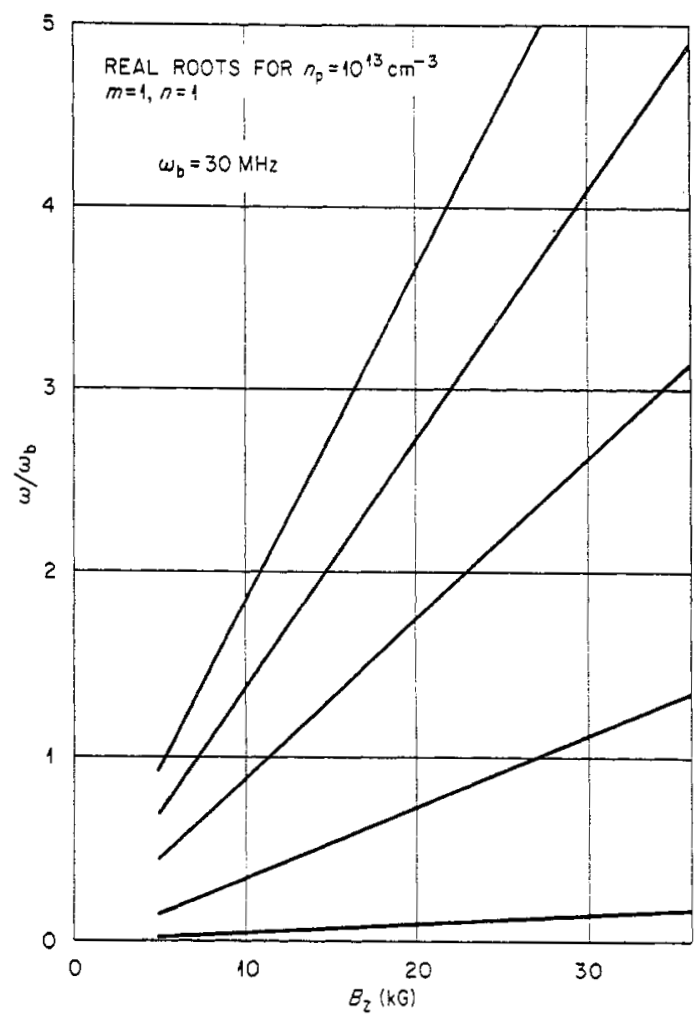

Fig. 3,- Roots of the fixed boundary dispersion relation vs. $B_{z}$ with $n_{B}=0$ and at a fixed $n_{p}$.

purely real roots are possible and that these correspond to the Alfven waves of a cylindrical cavity.

In Figs 2 and 3 roots of the dispersion relation (66) are plotted as functions of plasma density (at fixed toroidal field) and toroidal field (at fixed plasma density). As may be seen, the frequencies do scale with the Alfven velocity; they vary linearly in magnetic field strength and are inversely proportional to the square root of the plasma density.

By examining the relative sizes of the terms in equation (71) with the beam density equal to zero, some approximate analystic results may be obtained to explain the curves of Figs 2 and 3. The exact dispersion relation is given by

$$
Z_{2} \rho_{a} T_{1}^{2} T_{2} \frac{J_{m}^{\prime}\left(\rho_{a} T_{2}\right)}{J_{m}\left(\rho_{a} T_{2}\right)}-Z_{1} \rho_{a} T_{1} T_{2}^{2} \frac{J_{m}^{\prime}\left(\rho_{a} T_{1}\right)}{J_{m}\left(\rho_{a} T_{1}\right)}+\frac{D P}{S+Q} m\left(Z_{1}-Z_{2}\right)=0
$$

With the beam density equal to zero and for plasma densities, toroidal fields, and minor radii typical of Tokamaks, the first term in the above equation is 4-5 orders of magnitude lower than the other two; also, $Z_{2}$ is several orders of magnitude less than $Z_{1}$. Thus (72) is adequately approximated by the dispersion relation

$$
\left.-\rho_{a} T_{1}\left(S-n_{\|}{ }^{2}\right) J_{m}{ }^{\prime}\left(\rho_{a} T_{1}\right)+m D J_{m}\right)\left(\rho_{a} T_{1}\right)=0 .
$$

Also, for the frequency range of interest $\left(\omega \leq \Omega_{c i}\right)$ the following approximations 
may be made:

$$
\begin{aligned}
S-n_{\|}^{2} & \simeq S, \\
T_{1}^{2} & \simeq c^{2} / v_{A}^{2}, \\
D / S & \simeq-\omega / \Omega_{i} .
\end{aligned}
$$

Defining the quantities

$$
\begin{aligned}
& x=a \omega / v_{A}, \\
& \delta=c / a \omega_{p i},
\end{aligned}
$$

The above dispersion relation may be written as

$$
J_{m-1}(x)+m\left(\delta-\frac{1}{x}\right) J_{m}(x)=0 .
$$

Zeros of this equation as a function of the parameter $\delta$ have been calculated numerically in SPONG (1976) for $m=1$ and $m=2$. It was found that for values of $\delta$ characteristic of Tokamaks, the zeros of (76) do not depend strongly on $\delta$ and are approximately equal to the zeros of $J_{m-1}$. Thus the roots of (72) are $\omega \simeq$ $j_{m-1, s} v_{A} / a$.

\section{NUMERICAL RESULTS FOR GROWTH RATES}

Unstable roots for the fixed boundary kink mode dispersion relation have been calculated over a range of parameters which are typical of Tokamak strong runaway discharges. These results are presented and discussed in this section. The roots of (71) were obtained by using the Cauchy root-finding subroutine developed by BEASLEY and MEIER (1974). Some care had to be taken in solving equation (71) due to the branch-cut present near the low frequency roots from the square root involved in computing $T_{1}$. This may be avoided, however, by noting that the second term of (71) is an even function of $T_{1}$ and thus may be written in a form which depends only on $T_{1}^{2}$.

\section{Dependence on beam energy}

In Figs 4-7 growth rates and real frequencies are plotted for beam energies of $250 \mathrm{keV}, 500 \mathrm{keV}, 1 \mathrm{MeV}$ and $14.5 \mathrm{MeV}$. The beam is assumed to carry $100 \%$ of

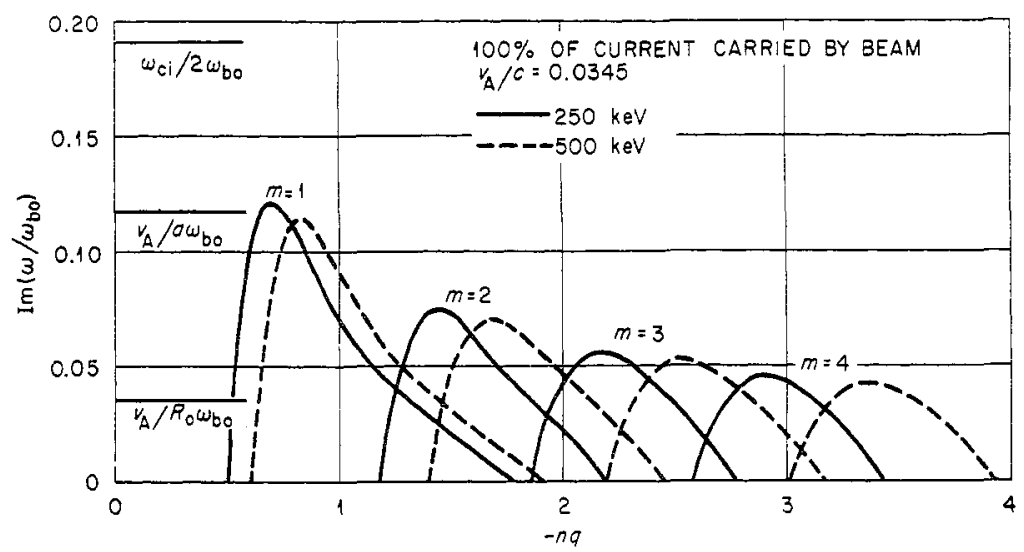

FIG. 4.-Growth rates for $m=1,2,3,4 ; n=-1$ kink modes vs. $n q$ and beam energy $(250,500 \mathrm{keV})$ for $v_{\mathrm{A}} / c=0.0345$. 


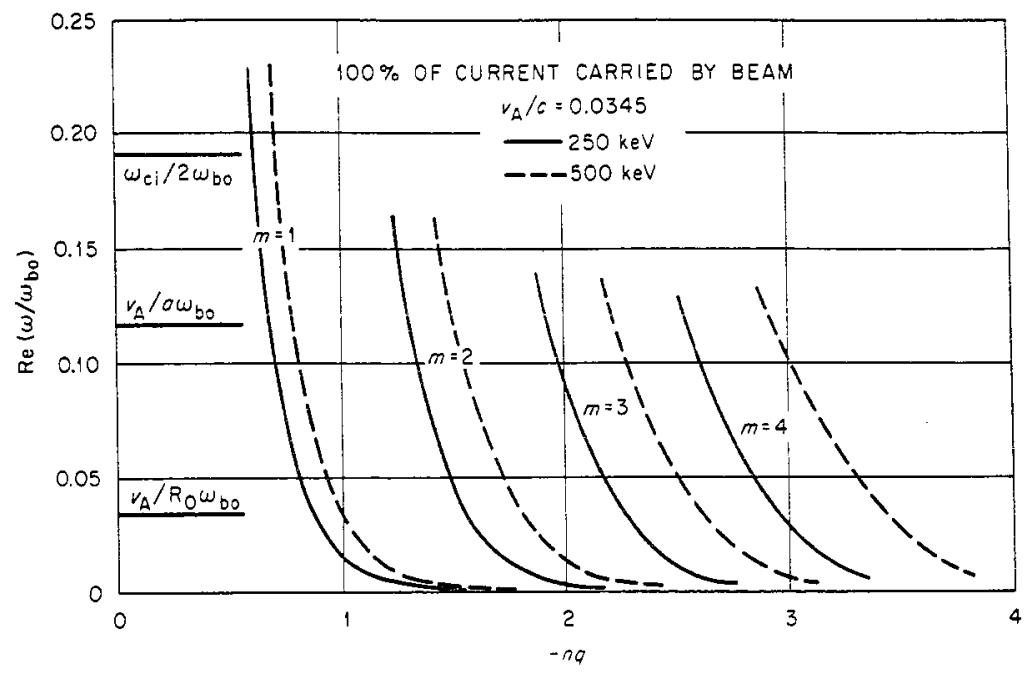

Fig 5.-Real part of the unstable roots for $m=1,2,3,4, ; n=-1$ kinks vs. $n q$ and beam energy $(250,500 \mathrm{keV})$ for $v_{A} / c=0.0345$.

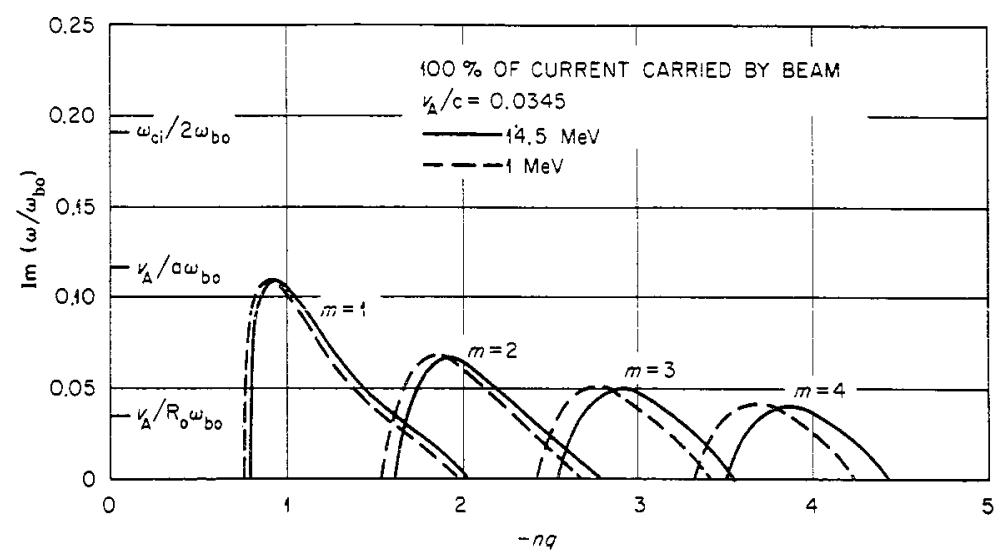

FIG. 6.-Growth rates for $m=1,2,3,4 ; n=-1$ kink modes vs. $n q$ and beam energy $(1,14.5 \mathrm{MeV})$ for $v_{\mathrm{A}} / c=0.0345$.

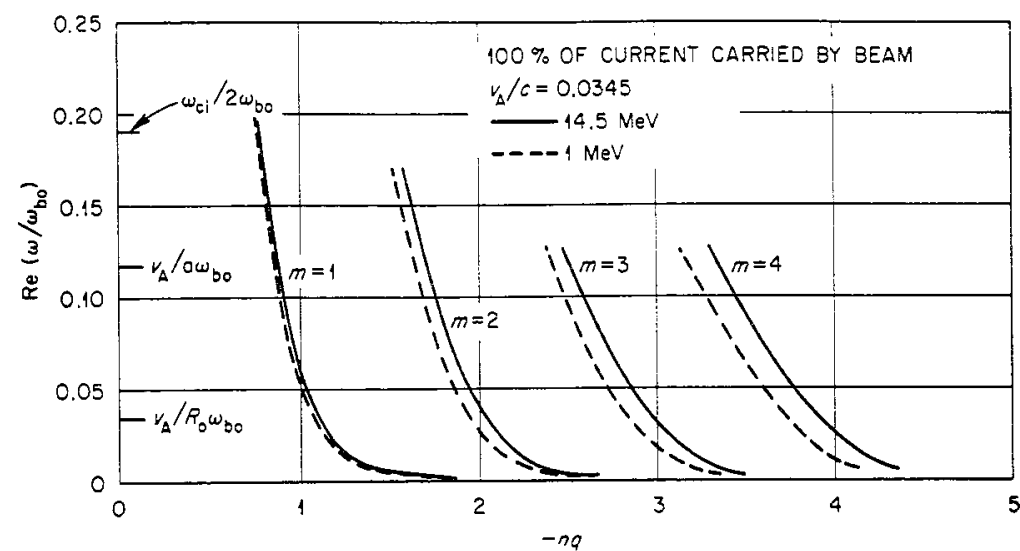

Fig. 7.-Real part of the unstable roots for $m=1,2,3,4 ; n=-1$ kink modes vs. $n q$ and beam energy $(1,14.5 \mathrm{MeV})$ for $v_{\mathrm{A}} / c=0.0345$. 
the current and the toroidal field and plasma density are $15 \mathrm{kG}$ and $10^{13} \mathrm{~cm}^{-3}$. Fairly slight differences are present between the $1 \mathrm{MeV}$ and $14.5 \mathrm{MeV}$ cases, but large changes are apparent in going from $250 \mathrm{keV}$ to $500 \mathrm{keV}$ to $1 \mathrm{MeV}$. Increasing the energy seems to shift the growth rate curves to the right on the q-axis, though not enough to change the shape substantially. This effect is particularly noticeable for the higher $m$ numbers.

\section{Dependence on $v_{\mathrm{A}} / \mathrm{c}$}

Growth rates and real frequencies are plotted in Figs 8 and 9 for $v_{A} / c=0.058$, 0.042 and 0.034 . These correspond to a plasma density of $10^{13} \mathrm{~cm}^{-3}$ and toroidal fields of 15,18 and $25 \mathrm{kG}$, respectively. The beam is assumed to carry all of the toroidal current and has an energy of $1 \mathrm{MeV}$. The growth rates appear to be monotonically increasing with $v_{A} / c$, as is the case in ideal MHD. It may be noted that the upper value of $q$ where the threshold occurs is relatively independent of $v_{A} / c$, whereas the lower threshold is not. The lower marginal point goes to lower values of $q$ as $v_{\mathrm{A}} / \mathrm{c}$ is increased.

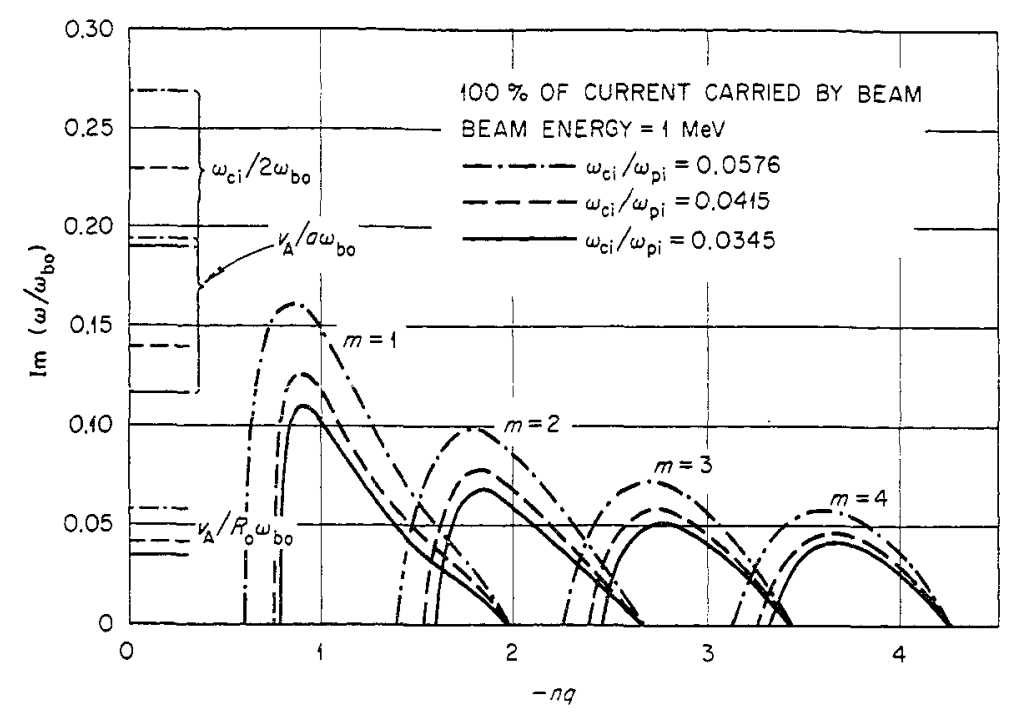

FIG. 8. Growth rates for $m=1,2,3,4 ; n=-1$ kink modes vs. $n q$ and $\omega_{\mathrm{ci}} / \omega_{\mathrm{pi}}$ for $1 \mathrm{MeV}$ beam energy.

\section{CONCLUSIONS}

The purpose of this work, as discussed in Section 1, has been to examine the macroscopic stability features of toroidal relativistic beams. A normal mode approach has been taken using a cylindrical geometry with period boundary conditions on the perturbed fields to simulate a torus. The following new features have been included: first the background plasma model has been extended to include frequencies which are not small relative to the ion gyrofrequency. This was motivated by the fact that high energy electrons circulate around flux surfaces at frequencies of the order of $\Omega_{c i}$. Macroscopic modes associated with this component are thus not necessary limited to frequencies $\ll \Omega_{c i}$ as is the case in ideal MHD. Second, both the transverse and longitudinal dynamics of the beam 


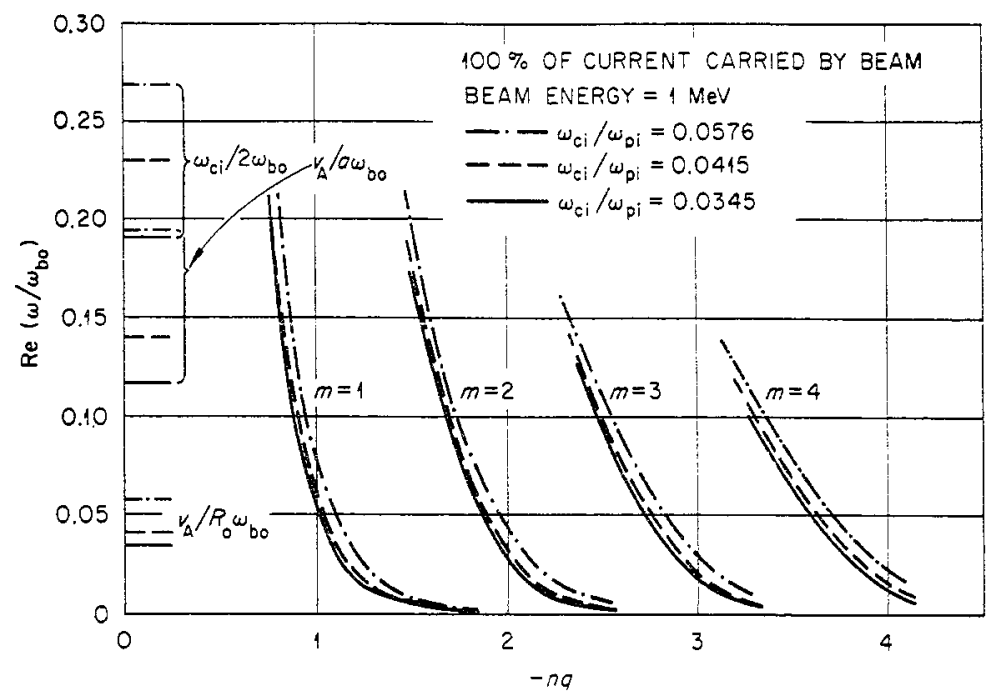

FiG. 9.-Real part of the unstable roots for $m=1,2,3,4 ; n=-1$ kink modes vs. $n q$ and $\omega_{c i} / \omega_{p i}$ for $1 \mathrm{MeV}$ beam energy.

electrons are included, whereas previous work has primarily treated the transverse dynamics.

In general, the results for growth rates of kink modes show that maxima occur near the rational magnetic surfaces-as is the case in ideal MHD. However, just to the inside of the rational surface, it was usually observed that the real part of the unstable root rose to high values (up to half of the ion gyrofrequency) while the growth rate dropped rapidly to zero, resulting in stable regions just to the inside of the rational surfaces. We interpret this characteristic as follows. Near the rational $q$ surfaces, the high energy electron orbits are closely aligned with the helical mode structure of the instability which typically grows on a much slower time scale than the drift timescale associated with the fast electrons. Just to the inside of rational $q$ surfaces, the fast electron orbits are no longer in resonance with the instability mode structure. This component can thus connect regions of positive and negative current perturbation just inside the rational surface. Such imbalances may be shorted out by the fast electrons since their poioidal drift frequency is substantially higher that the growth rate of the current perturbations.

A second feature which was observed in these calculations was that the term in the dispersion relation which arises from the longitudinal electron inertia (the first term in equation 66) is typicaily 4-5 orders of magnitude less than the other terms in the dispersion relation. Thus, at least for the fixed boundary model, it appears that the longitudinal dynamics of the relativistic electrons is not an important factor in determining the growth rates for the instabilities examined here.

In closing, it is noted that this treatment, as well as the previous work (LEE, 1973; LOVELACE, 1976), has been concerned primarily with cylindrical models. The influence of toroidal geometry has been taken into account only by requiring periodic boundary conditions on the perturbed fields. However, there are two other effects which enter in with toroidal geometry and merit further consideration. One is the coupling between various order $m$ modes caused by poloidal variations in beam equilibrium properties. A second effect is large displacements 
of the single particle orbits off of flux surfaces when energies become relativistic. These effects are discussed in further detail along with orbit calculations for relativistic electrons in Tokamaks elsewhere (SPONG, 1976).

Acknowledgements-This research was supported by the United States Energy Research and Development Administration and through a Graduate Participantship from Oak Ridge Associated Universities.

\section{REFERENCES}

Alikaev V. V., Arsenev Y. I., Bobrovskil G. A., Kouorariev A. A. and Razumova K. A. (1973) MATT-TRANS-111. Princeton, New Jersey.

Alikaev V. V., Razumova K. A. and Sokolov Yu. A. (1975) Sov. J. Plasma Phys. 1, 303.

Benson J., ECKer B. and BALEy V. (1974) Phys. Rev. Lett. 33, 574.

BeAsley C. O. and MeIER H. K. (1974 ORNL/TM-4588. Oak Ridge, Tennessee.

Bludman S. A., Watson K. M. and Rosendluth M. N. (1960) Physics Flüids 3, 747.

Costly A. E., Hastie R. J., Paul J. W. M. and Chamberlain J. (1974) Phys. Rev. Lett. 33, 758.

Gilad P., Kusse B. R. and Lockner T. R. (1974) Phys. Rev. Lett. 33, 1275.

Guest G. E., Hedrick C. L. and Nelson D. B. (1975) Physics Fluids 18, 871.

Hamier D. A. and Pa Padoupolous K. (1975) Nucl. Fusion 15, 977.

Jornson C. C. (1965) Field and Wave Electrodynamics. McGraw-Hill, New York.

KNOEPFel H., SpONG D. A. and ZweBen S. J. (1977) Physics Fluids 20, 511.

Lee E. P. (1973) Physics Fluids 16, 1072.

LOVELACE R. (1976) Physics Fluids 19, 723.

Mohri A., Masuzaki M., Tsuzuki T. and IkUta K. (1975) Phys. Rev. Lett. 34, 574.

Spong D. A., Clarke J. F., Rome J. A. and Kammash T. (1974) Nucl. Fusion 14, 397.

SPONG D. A. (1976) Ph.D Dissertation. Univ. of Michigan, Ann Arbor, Michigan; ORNL/TM-5147, Oak Ridge, Tennessee.

SwaIN D. W., Miller P. A. and Widner M. M. (1975) SAND 75-0214, Albuquerque, New Mexio.

Vlasenkov V. S., Leonov V. M., Merezhkin V. G. and Mukohvatov V. S. (1973) Nucl. Fusion 13, 509.

\section{APPENDIX A}

Perturbed beam currents for a beam with energy spread

As was indicated in Section 3 , an alternate formulation must be employed in calculating perturbed beam currents when energy spread is present in the relativistic beam. This is presented in the following and is based on the relativistic Vlasov equation.

$$
\frac{\partial f}{\partial t}+\mathbf{v} \cdot \nabla f+\frac{e}{m_{0}}\left[\mathbf{E}+\frac{1}{c}(\mathbf{v} \times \mathbf{B})\right] \cdot \frac{\partial f}{\partial \mathbf{p}}=0 .
$$

where $\overrightarrow{\mathbf{p}}=\gamma m_{0} \mathbf{v}$ and $\gamma=\left(1-v^{2} / c^{2}\right)^{-1 / 2}$.

The above equation is linearized by assuming $f=f_{0}+\delta f$ where $f_{0}$ is an equilibrium distribution function. $\delta f$ is then obtained by integrating over the characteristic (unperturbed orbits).

$$
\delta f=\frac{e}{m_{0}} \int_{-\infty}^{t} \mathrm{~d} t^{\prime}\left[\mathbf{E}+\frac{\mathbf{v} \times \mathbf{B}}{c}\right] \cdot \frac{\partial f_{0}}{\partial \mathbf{p}} .
$$

The equilibrium distribution function $f_{0}$ depends only on the three constants of the motion which are given below.

$$
\begin{aligned}
& H=\gamma m_{0} c^{2} \\
& P_{z}=\gamma m_{0} v_{z}+\frac{e}{c} A_{z}, \\
& L_{\theta}=\gamma m_{0}\left(x v_{y}-y v_{x}\right)+\frac{e}{c} r A_{\theta} .
\end{aligned}
$$

Expanding $\partial f_{0} / \partial \mathbf{p}$ by the chain rule allows one to express $\partial f$ as follows.

$$
\delta f=\frac{\partial f_{0}}{\partial H} \delta H+\frac{\partial f_{0}}{\partial P_{z}} \delta P_{z}+\frac{\partial f_{0}}{\partial L_{\theta}} \delta L_{\theta}
$$


where

$$
\begin{aligned}
& \delta H=\int_{-\infty}^{t} \mathrm{~d} t^{\prime}(\mathbf{v} \cdot \delta \mathbf{F}), \\
& \delta P_{z}=\int_{-\infty}^{t} \mathrm{~d} t^{\prime} \delta F_{z}, \\
& \delta L_{\theta}=\int_{-\infty}^{t} \mathrm{~d} t^{\prime}\left(x \delta F_{y}-y \delta F_{z}\right) .
\end{aligned}
$$

We shall consider equilibrium distribution functions here which depend only on $H$ and $P_{z}$, i.e. $f_{0}=f_{0}\left(H, P_{z}\right)$. Using the unperturbed relativistic beam orbits given in Section 2 , the time history integrals given in (A.4) may be performed. Assuming that $\omega_{+} \gg \omega_{-}$(gyrofrequency is much faster than the transit frequency around the torus) results in the equations given below for $\delta P_{z}$ and $\delta H$.

$$
\begin{gathered}
\delta P_{z}=\frac{i e E_{z}}{m_{0}\left(\omega-\omega_{b r} n q-n \omega_{-}\right)} . \\
\delta H=\frac{i e\left(v_{z} E_{z}-r \omega_{2} E_{\theta}\right)}{m_{0}\left(\omega-\omega_{b r} n q-m \omega_{-}\right)}-\frac{i e}{m_{0} \omega_{-}}\left(v_{\theta} E_{r}-v_{r} E_{\theta}\right),
\end{gathered}
$$

The perturbed relativistic beam current is then given by the following equation.

$$
\delta J=-e \int_{0}^{\infty} p_{\perp} p d_{\perp} \int_{0}^{\infty} \operatorname{vd} p_{\|}\left[\delta P_{z} \frac{\partial f_{0}}{\partial P_{z}}+\delta H \frac{\partial f_{0}}{\partial H}\right]
$$

Substituting $\delta P_{z}$ and $\delta H$ from (A.6) and (A.7) into (A.8) and performing an integration by parts on the first term, one obtains the result given below.

$$
\begin{aligned}
\delta J= & -\omega_{p b}^{2} \int_{0}^{\infty} \rho_{\perp} \mathrm{d} \rho_{\perp} \int_{-\infty}^{\infty} \mathrm{d} p_{\|} \vee\left\{\frac{1}{m_{0}}\left(\omega-\omega_{b r} n q-m \omega_{-}\right)^{-1},\right. \\
& \left.\times \frac{f_{0}}{c \gamma^{3}} E_{z}+\frac{1}{c^{2}}\left(v_{z} E_{z}-r \omega_{b} E_{\theta}\right) \frac{\partial f_{0}}{\partial \gamma}, \quad-\frac{1}{\Omega_{e} c^{2}}\left(v_{\theta} E_{r}-v_{r} E_{\theta}\right) \frac{\partial f_{0}}{\partial \gamma}\right\} .
\end{aligned}
$$

It is more convenient from this point on to convert the above integral to an integration over $v_{\|}$and $\gamma$. The Jacobian of this transformation is given by the following.

$$
p_{\perp} \mathrm{d} p_{\perp} \mathrm{d} p_{\|}=p_{\perp}|J| \mathrm{d} v_{\|} \mathrm{d} \gamma
$$

where

$$
|J|=m_{0}^{2} v_{\perp}^{2} \gamma
$$

In performing the integrations indicated in (A.9) we shall assume a delta function distribution in $v_{\|}$ with a spread allowed in the energy dependence, i.e. $f_{0}\left(v_{\|}, \gamma\right)=\delta\left(v_{\|}-v_{0}\right) F(\gamma)$ where $v_{0} \approx c$. If $\gamma_{0}=\left(1-v_{0}^{2} / c^{2}\right)^{-1 / 2}$, then

$$
p_{\perp}|J|=m_{0} c^{2}\left(\frac{\gamma^{2}}{\gamma_{0}^{2}}-1\right)
$$

We shall consider a step function dependence for $F(\gamma)$.

$$
F(\gamma)= \begin{cases}0 & \gamma<\gamma_{0}, \\ K & \gamma_{0}<\gamma<\gamma_{m}, \\ 0 & \gamma>\gamma_{m} .\end{cases}
$$

where $K$ is is a normalization constant. 
Substituting the above distribution function into (A.9) then results in the following perturbed currents.

$$
\begin{gathered}
\delta J_{r}=-\frac{2 i \omega_{p b}{ }^{2}}{m_{0}} \chi\left(\gamma_{0}, \gamma_{m}\right) \frac{\gamma_{0}-\gamma_{m}}{\omega_{+} \gamma_{0}^{2} c^{2}} E_{\theta} . \\
\delta J_{\theta}=\frac{2 i \omega_{p b}{ }^{2}}{m_{0}} \chi\left(\gamma_{0}, \gamma_{m}\right) \frac{\gamma_{0}-\gamma_{m}}{\omega_{+} \gamma_{0}{ }^{2} c^{2}} E_{r} \\
\delta J_{z}=-\frac{i \omega_{p b}{ }^{2}}{m_{0}} \chi\left(\gamma_{0}, \gamma_{m}\right)\left[\frac{I_{1}}{\gamma_{0}{ }^{2}} E_{z}-I_{2} E_{z}-\frac{2}{\gamma_{0}{ }^{2} c^{2}}\left(c E_{z}-r \omega_{b r} E_{\theta}\right) I_{3}\right]
\end{gathered}
$$

where

$$
\begin{gathered}
\chi\left(\gamma_{0}, \gamma_{m}\right)=\frac{3}{2 \pi \gamma_{0}}\left[\frac{\gamma_{m}{ }^{3}}{\gamma_{0}^{3}}-3 \frac{\gamma_{m}}{\gamma_{0}}+2\right]^{-1} . \\
I_{1}=-\left[\omega-\omega_{b r}(n q+m)\right]^{-1} \ln \left\{\frac{\gamma_{0}\left[\omega-\omega_{b r}(n q+m)-\varepsilon m \omega_{b r} \gamma_{m}\right]}{\gamma_{m}\left[\omega-\omega_{b r}(n q+m)-\varepsilon m \omega_{b r} \gamma_{0}\right]}\right\} \\
I_{2}=\left[\omega-\omega_{b r}(n q+m)\right]^{-1}\left\{\frac{1}{2}\left(\frac{1}{\gamma_{0}^{2}}-\frac{1}{\gamma_{m}^{2}}\right)+\frac{\varepsilon m \omega_{b r}}{\omega-\omega_{b r}(n q+m)}\left(\frac{1}{\gamma_{0}}-\frac{1}{\gamma_{m}}\right)\right\} \\
-\frac{\varepsilon^{2} m^{2} \omega_{b r}^{2}}{\left[\omega-\omega_{b r}(n q+m)\right]^{3}} \ln \left[\frac{\gamma_{0}\left[\omega-\omega_{b r}(n q+m)-\varepsilon \omega_{b r} m \gamma_{m}\right]}{\gamma_{m}\left[\omega-\omega_{b r}(n q+m)-\varepsilon \omega_{b r} m \gamma_{0}\right]}\right] \\
I_{3}=\frac{\gamma_{0}-\gamma_{m}}{\varepsilon m \omega_{b r}}-\frac{\omega-\omega_{b r}(n q+m)}{\varepsilon^{2} m^{2} \omega_{b r}^{2}} \ln \left[\frac{\omega-\omega_{b r}(n q+m)-\varepsilon \omega_{b r} m \gamma_{m}}{\omega-\omega_{b r}(n q+m)-\varepsilon \omega_{b r} m \gamma_{0}}\right] \\
\varepsilon=2 \omega_{b r} \Omega_{e} .
\end{gathered}
$$

